\title{
Tratamiento quirúrgico conservador en un paciente con invaginación yeyuno-yeyunal secundaria a síndrome de Peutz-Jeghers. Reporte de un caso
}

\author{
Conservative surgical treatment in a patient with jejunal- \\ jejunal intussusception secondary to Peutz-Jeghers \\ syndrome. Case rport.
}

Rodrigo Ugalde-Resano, ${ }^{1}$ Irina Jeanette Bercholc-Urinowsky, ${ }^{2}$ Luis Enrique Escalona-Ramírez, ${ }^{1}$ Juan Miguel Blanco-Torres, ${ }^{1}$ Miguel Ángel Paz-Muñoz, ${ }^{3}$ Raúl Escamilla-Roque, ${ }^{4}$ Octavio Bailón-Uriarte ${ }^{4}$

\begin{abstract}
Resumen
INTRODUCCIÓN: el síndrome de Peutz-Jeghers es una enfermedad autosómica dominante, poco frecuente, caracterizada por hiperpigmentación mucocutánea y pólipos gastrointestinales; estos últimos causantes de obstrucciones intestinales recurrentes, secundarias a invaginación.

PRESENTACIÓN DEL CASO: adolescente femenina, de 13 años, que acudió a urgencias debido a un cuadro de dolor abdominal intenso, con un cuadro de obstrucción intestinal secundario a invaginación yeyuno-yeyunal. Siete años antes tuvo un cuadro clínico similar que desapareció espontáneamente. A la exploración física se observaron lesiones hiperpigmentadas en el labio inferior y anemia microcítica e hipocrómica con los que se integró el diagnóstico de síndrome de Peutz-Jeghers. Debido a que en la tomografía axial computada de abdomen se evidenció invaginación intestinal se realizó una laparotomía exploradora, con el hallazgo de dos pólipos hamartomatosos de 2 y $3 \mathrm{~cm}$ de diámetro a $15 \mathrm{~cm}$ del ligamento de Treitz. Se trató la invaginación intestinal y, posteriormente, se efectuaron enterotomía y polipectomía. Mediante enteroscopia transoperatoria se descartaron otras alteraciones. Luego de tratar el cuadro clínico la paciente fue dada de alta del hospital en buenas condiciones.

CONCLUSIÓN: la invaginación intestinal en adolescentes es una situación excepcional que requiere un alto índice de sospecha para enfermedades polipósicas. Se reporta un caso de invaginación intestinal que se trató existosamente con cirugía conservadora, sin necesidad de resecciones intestinales que, a largo plazo, condicionan síndrome de intestino corto: uno de los principales problemas a los que se enfrentan estos pacientes.

PALABRAS CLAVE: Peutz-Jeghers, pólipos gastrointestinales, obstrucción intestinal recurrente, dolor abdominal agudo, yeyuno-yeyunal, adolescentes.

\section{Abstract}

INTRODUCTION: Peutz-Jeghers syndrome is an uncommon autosomal dominant disease, characterized by mucocutaneous hyperpigmentation and gastrointestinal polyps, which are the cause of recurrent intestinal obstructions, secondary to invagination. This case provides evidence to consider more conservative surgical treatments and prevent these patients from being constantly subjected to extensive bowel resections, which in many cases, can condition short bowel disease.

CASE PRESENTATION: A 13-year-old female patient who came to the emergency room with acute abdominal pain due to intestinal obstruction secondary to jejuno-jejunal intussusception. 7 years ago, the patient reported similar clinical manifestations that solved spontaneously. Physical examination showed hyperpigmented lesions on the lower lip, and microcytic hypochromic anemia; Peutz-Jeghers syndrome was established. Intestinal intussusception was evidenced by computed axial tomography of the abdomen, so exploratory laparotomy was performed, with the finding of two hamartomatous
\end{abstract}

\footnotetext{
${ }^{1}$ Departamento de Cirugía general, Hospital Central Norte PEMEX.

${ }^{2}$ Departamento de Pediatría, Hospital Central Sur de Alta Especialidad PEMEX ${ }^{3}$ Departamento de Imagenología, Hospital Central Norte PEMEX.

${ }^{4}$ Departamento de Cirugía general, Hospital Regional de Minatitlán PEMEX.
}

Recibido: 30 de mayo 2017

Aceptado: 14 de julio 2017

Correspondencia Octavio Bailón Uriarte dr_bailonu@live.com

Este artículo debe citarse como Ugalde-Resano R, Bercholc-Urinowsky IJ, Escalona-Ramírez LE, Blanco-Torres JM, Paz-Muñoz MA, Escamilla-Roque R, BailónUriarte $\mathrm{O}$. Tratamiento quirúrgico conservador en un paciente con invaginación yeyuno-yeyunal secundaria a síndrome de PeutzJeghers. Reporte de un caso. Acta Pediatr Mex. 2018;39(3):244-249. 
polyps 2 and $3 \mathrm{~cm}$ in diameter at $15 \mathrm{~cm}$ of the Treitz ligament. Intestinal intussusception was resolved with subsequently, enterotomy and polypectomy. Other alterations were ruled out by trans-operative enteroscopy. At the resolution of clinical manifestations, the patient was discharged from the hospital in good clinical conditions.

CONCLUSION: Intestinal intussusception in adolescents is an exceptional situation, which requires a high index of suspicion for polysposis diseases. We present a case of intestinal intussusception, successfully resolved with conservative surgical treatment, without requiring intestinal resections, which can condition short bowel syndrome in the long-term, one of the major problems these patients face.

KEYWORDS: Peutz-Jeghers; gastrontestinal polyps; recurrent intestinal obstructions; acute abdominal pain; jejuno jejunal; adolescents.

\section{INTRODUCCIÓN}

El síndrome de Peutz-Jeghers, descrito por primera vez en 1921 por Peutz ${ }^{1}$ y Jeghers en 1949, ${ }^{2}$ es una enfermedad autosómica dominante, infrecuente, caracterizada por hiperpigmentación mucocutánea y pólipos gastrointestinales, ${ }^{3}$ estos últimos son los causantes de obstrucciones intestinales recurrentes, secundarias a invaginación. El tratamiento convencional consiste en resecciones intestinales amplias que, eventualmente, pueden condicionar la aparición del síndrome de intestino corto. ${ }^{4}$

Enseguida se reporta un caso que fue tratado exitosamente de manera conservadora.

\section{CASO CLÍNICO}

Paciente femenina de 13 años, que acudió a urgencias debido a dolor abdominal intenso en el epigastrio, de 4 horas de evolución y vómito de contenido gastrobiliar. La madre refirió que la paciente tuvo un cuadro similar 7 años antes del actual, que remitió espontáneamente; negó enfermedades crónicas.

A la exploración física se encontró ansiosa y taquicárdica. En el labio inferior se observaron lesiones hiperpigmentadas, que aparecieron en la edad prescolar. (Figura 1) El abdomen se observó con perímetro incrementado, doloroso a la palpación, con predominio en el cuadrante

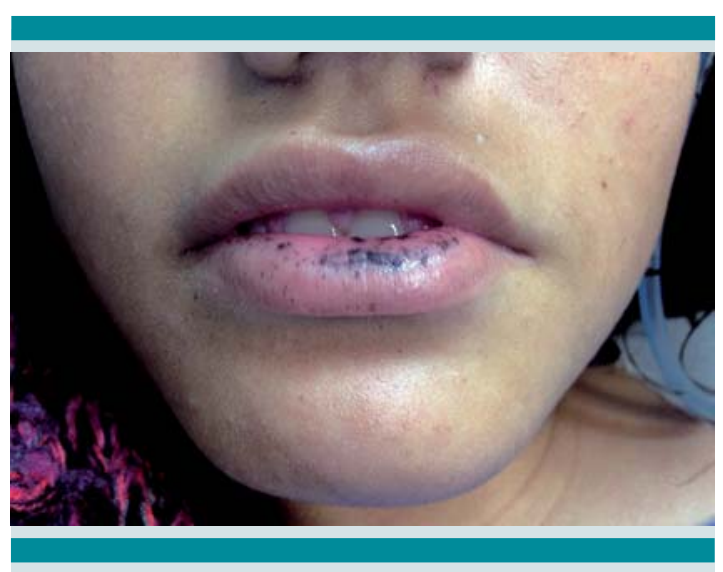

Figura 1. Lesiones hipercrómicas características del síndrome de Peutz-Jeghers.

superior izquierdo. Se palpó un tumor de 5 cm en relación con un asa distendida, en posición transversa, peristalsis incrementada en el cuadrante afectado. El resto de la exploración trascurrió normal. La biometría hemática reportó anemia microcítica e hipocrómica, con hemoglobina de $8.5 \mathrm{~g} / \mathrm{dL}$. El resto de los estudios de laboratorio se reportaron normales. (Cuadro 1)

El ultrasonido abdominal sugirió probable invaginación intestinal; posteriormente se corroboró con tomografía computada de abdomen, al observar el "signo de la rosquilla", imagen característica en diana, con bandas de diferente densidad concéntricas, en relación con las paredes del asa del intestino que se introducían dentro de su propia luz. (Figura 2) 
Cuadro 1. Estudios de laboratorio y valores de referencia en mujeres a partir de 12 años de edad

\begin{tabular}{|ccl|}
\hline \multicolumn{2}{|c|}{ Valores de referencia } & \multicolumn{1}{c|}{ Estudio } \\
\hline $8.1(4.5-13.5)$ & 7.9 & Leucocitos $\left(\times 10^{3} / \mu \mathrm{L}\right)$ \\
\hline $4.4(1.5-8.5)$ & 4.7 & Neutrófilos $\left(\times 10^{3} / \mu \mathrm{L}\right)$ \\
\hline $3.1(1.5-6.8)$ & 2.6 & Linfocitos $\left(\times 10^{3} / \mu \mathrm{L}\right)$ \\
\hline 0.4 & 0.5 & Monocitos $\left(\times 10^{3} / \mu \mathrm{L}\right)$ \\
\hline $4.1-5.10$ & 4.47 & Eritrocitos $\left(\times 10^{6} / \mu \mathrm{L}\right)$ \\
\hline $14(12.0)$ & 8.5 & Hemoglobina $(\mathrm{g} / \mathrm{dL})$ \\
\hline $41(37)$ & 28.9 & Hematócrito $(\%)$ \\
\hline $90(78)$ & $64.6 \mathrm{fL}$ & VMC (fL) \\
\hline $34(31.0)$ & 19 & HCM $(\mathrm{pg} / \mathrm{eritrocito})$ \\
\hline $150-350$ & 335 & Plaquetas $\left(\times 10^{3} / \mu \mathrm{L}\right)$ \\
\hline $13.8(12.7-16.1)$ & $14.7 \mathrm{seg}$ & TP $($ segundos $)$ \\
\hline $1.08(0.97-1.30)$ & 1.08 & INR \\
\hline $39.5(33.9-46.1)$ & $23.6 \mathrm{seg}$ & TTPa (segundos) \\
\hline & O Rh + & Hemotipo \\
\hline
\end{tabular}

VCM ( $\mathrm{fL}$ ): volumen corpuscular medio. HCM (pg/eritrocito): hemoglobina corpuscular media. TP (segundos): tiempo de protrombina. INR: International Normalized Ratio. TTPa (segundos): tiempo parcial de tromboplastina activada. Referencias tomadas y adaptadas de: Engorn B, Flerlage J. Table 14-1: Age-Specific Blood Cell Indices y Table 14-6: Age-Specific Leukocyte Differential de The Harriet Lane Handbook, Twentieth Edition, Elsevier, 2014; y de Prevención, diagnóstico y tratamiento de la anemia por deficiencia de hierro en niños y adultos. Guía de Práctica Clínica. Catálogo maestro de guías de práctica clínica: IMSS-415-10 http://www.cenetec.salud.gob.mx/descargas/gpc/CatalogoMaestro/415_IMSS_10_Anemia_def_hierro_May2a/ GRR_IMSS_415_10.pdf

En la laparotomía exploradora se observó la invaginación yeyuno-yeyunal (Figura 3) que se trató mediante taxis de intestino delgado, liberando $110 \mathrm{~cm}$ de asa intestinal. Debido a que se identificaron dos tumores intraluminales de 2 y $3 \mathrm{~cm}$ de diámetro a 15 centímetros del ligamento de Treitz (Figura 4) se efectuó enterotomía y posterior polipectomía de los mismos. (Figura 5) Después se practicó una enteroscopia transoperatoria y no se encontraron pólipos ni otras lesiones, por lo que se concluyó el procedimiento sin accidentes ni incidentes.
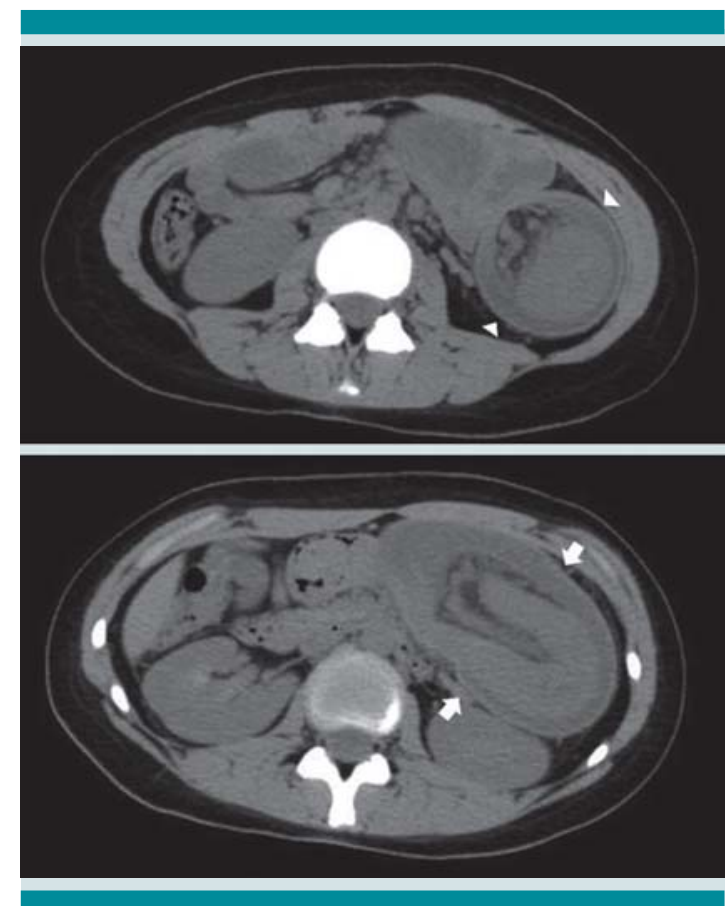

Figura 2. En la parte superior se observa una imagen clásica del signo de la rosquilla (punta de flecha). La imagen inferior se caracteriza por bandas de diferente densidad en relación con las paredes del asa del intestino que se introduce dentro de su propia luz (flechas).

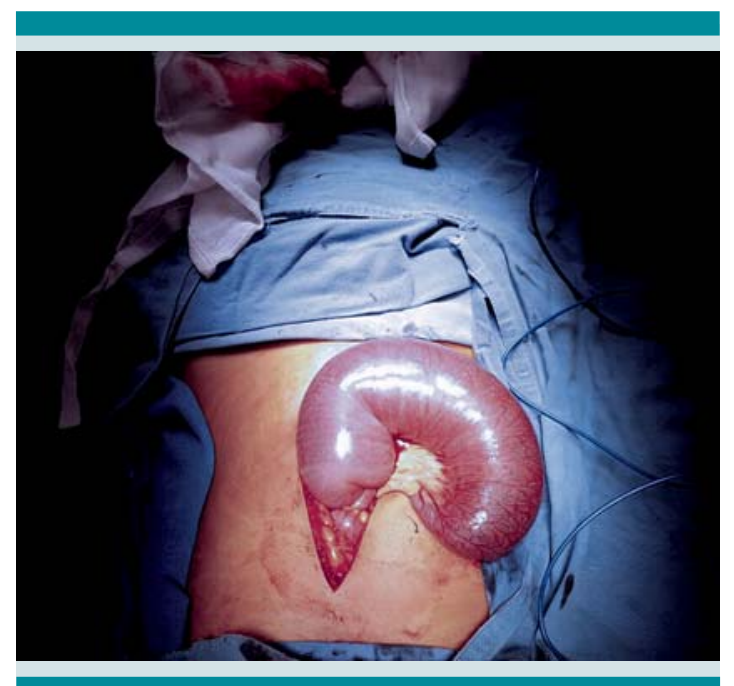

Figura 3. Laparotomía exploradora donde se observa la invaginación intestinal sin datos de sufrimiento. 


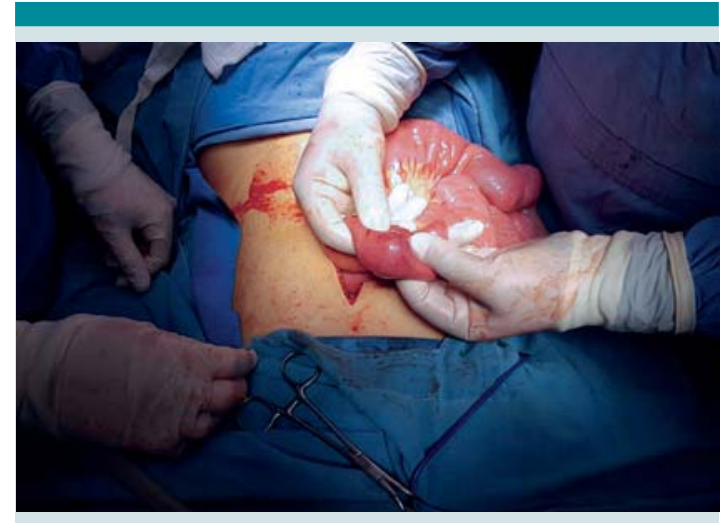

Figura 4. Pólipo intraluminal identificado por palpación a $15 \mathrm{~cm}$ del ligamento de Treitz.

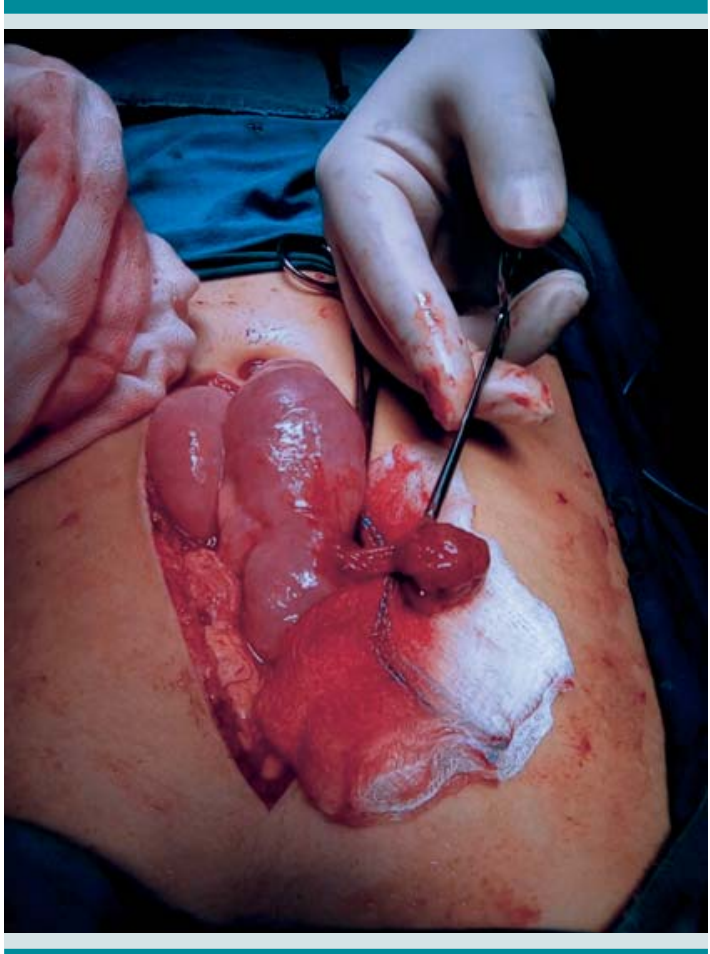

Figura 5. Pólipo pediculado identificado por enterotomía transoperatoria.

La evolución posoperatoria fue favorable, solo requirió transfusión de un paquete globular. Al cuarto día posoperatorio inició con dieta

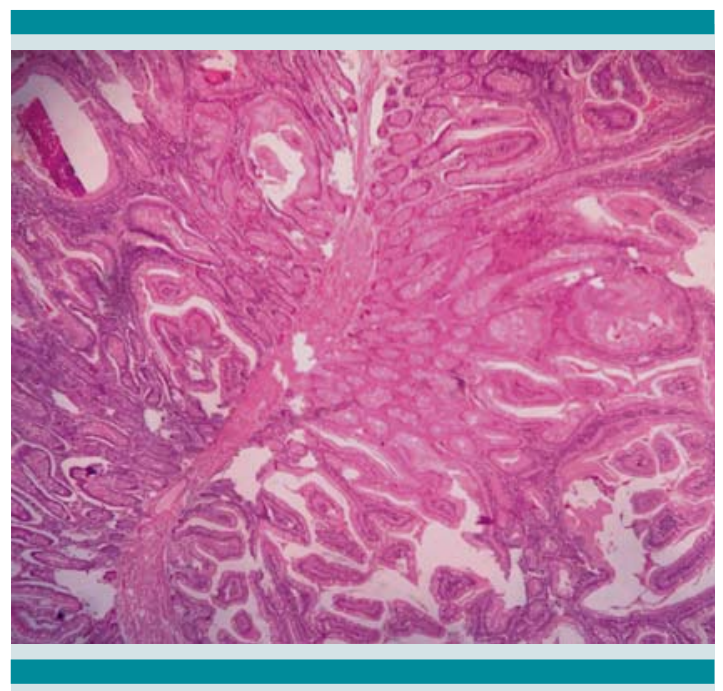

Figura 6. Histología de pólipo hamartomatoso. Se observa crecimiento aberrante de tejido normal con fibras de músculo liso en apariencia circunvolucionada. Mucosa intestinal con epitelio columnar absortivo, células de Paneth, argentafines y caliciformes. Sin displasia.

líquida y se dio de alta del hospital al quinto día de posoperada con adecuada tolerancia a la alimentación por vía oral. El reporte histopatológico fue de pólipos hamartomatosos, sin malignidad (Figura 6).

Con base en la historia clínica, la exploración física y el reporte de patología se integró el diagnóstico de síndrome de Peutz-Jeghers. La madre negó antecedentes familiares de poliposis o de enfermedades relacionadas; a pesar de ello se envió a consejería genética.

En la actualidad la paciente permanece asintomática y en seguimiento en la consulta externa. La colonoscopia no evidenció pólipos y se espera efectuar estudios endoscópicos seriados cada dos años para continuar su vigilancia. La información anónima y las imágenes de este documento fueron previamente autorizadas por la paciente y su madre, con fines estrictamente académicos. 


\section{DISCUSIÓN}

El síndrome de Peutz-Jeghers es un padecimiento raro, con incidencia incluso de 1 en 200,000 recién nacidos vivos, afecta por igual a uno y otro sexo. ${ }^{5} \mathrm{El}$ defecto se localiza en el gen supresor tumoral STK11(LKB1) localizado en el cromosoma 19. Se estima que 10 a $20 \%$ de los casos se manifestarán como mutaciones de novo, ${ }^{6}$ como el caso de la paciente aquí expuesto. Además, su penetrancia es variable, lo que significa que las manifestaciones clínicas también lo son. ${ }^{7}$

Las principales manifestaciones de los pacientes con síndrome de Peutz-Jeghers son: obstrucción intestinal, sangrado de tubo digestivo, anemia crónica e invaginación intestinal. El riesgo de invaginación intestinal aumenta con la edad del paciente, quizá secundario al tamaño de los pólipos y su crecimiento a través del tiempo; la incidencia máxima es en la edad adulta. ${ }^{8,9} \mathrm{En}$ el adolescente con invaginación intestinal debe sospecharse la posibilidad de una enfermedad polipósica, entre ellas: poliposis familiar, síndrome de Gardner y poliposis juvenil. Las tres se expresan con cuadros clínicos similiares. ${ }^{10}$

El 30\% de los pacientes con síndrome de PeutzJeghers a los 10 años de edad habrá requerido una laparotomía exploradora para tratar la invaginación intestinal ${ }^{11} \mathrm{y}$, posteriormente, una amplia resección intestinal para remover el mayor número de pólipos, que tienen un alto riesgo de recurrencia y de malignidad. Estas resecciones amplias pueden tener como consecuencia síndrome de intestino corto. ${ }^{12}$

En una cohorte de Van Lier y colaboradores, en la que se incluyeron 110 pacientes con síndrome de Peutz-Jeghers e invaginación intestinal, se reportó un riesgo acumulado de invaginación de $15 \%$ a los 10 años de edad, $50 \%$ a los 20 años, $65 \%$ a los 30 años, con incremento de $77 \%$ a los 40 años y de $84 \%$ a los 50 años. ${ }^{13}$ Aquí radica la importancia de continuar el seguimiento es- trecho y de realizar enteroscopias seriadas, con resección de los pólipos mayores de 15 mm. ${ }^{8,9}$

La prevención de complicaciones intestinales es un componente decisivo en la atención médica de estos pacientes. Lo ideal es iniciar la vigilancia entre los 8 y 10 años de edad y continuar en un intervalo de 2 años. ${ }^{14}$ Se recomienda efectuar estudios endoscópicos, como: gastroscopia, colonoscopia y enteroscopia con doble balón para realizar polipectomías en pólipos mayores de $15 \mathrm{~mm}$, con esto se consigue disminuir el riesgo de invaginación, obstrucción y resección quirúrgica. ${ }^{15}$ En la mayoría de los casos esto resulta difícil, sobre todo por falta de infraestructura o dificultades técnicas porque $80 \%$ de los pólipos se localizan en el yeyuno. ${ }^{16}$ Existen otras alternativas, como la cápsula endoscópica y la enterografía por resonancia magnética, pero estas modalidades se limitan solo a objetivos diagnósticos. ${ }^{14}$

A diferencia de los procedimientos diagnósticos y profilácticos, el tratamiento de las complicaciones agudas sigue siendo un reto para el cirujano porque ni la intervención quirúrgica o endoscópica son, por sí solas, una alternativa exitosa en todos los pacientes. ${ }^{15}$

La enteroscopia con doble balón no tiene indicación en casos de invaginación y obstrucción intestinal aguda, por eso la modalidad alternativa es el abordaje combinado, con resultados muy satisfactorios, como lo comentan Oncel y colaboradores, ${ }^{9}$ quienes Ilevaron a cabo un estudio comparativo en el que un primer grupo de pacientes fue tratado con un procedimiento combinado con laparotomía y enteroscopia asociado con polipectomía. Este grupo no requirió nuevas intervenciones, con un seguimiento de 21 años-paciente. El grupo control tratado solo con laparotomía y resección intestinal focalizada requirió 23 nuevos procedimientos en un seguimiento de 87 años-paciente, esto debido a la falta de visualización de los segmentos adyacentes, con pólipos no identificables al tacto. 
Ugalde-Resano R et al. Tratamiento quirúrgico conservador en síndrome de Peutz Jeghers

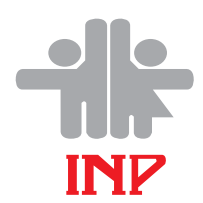

El procedimiento propuesto en este trabajo consiste en laparotomía exploradora con reducción manual de la invaginación, enteroscopia con polipectomía de los segmentos intestinales adyacentes; seguido o no de resección y anastomosis del segmento invaginado, según su viabilidad. Las complicaciones descritas de esta técnica son: íleo prolongado, distensión gaseosa y estenosis de la luz intestinal en los sitios de la polipectomía; sin embargo, se ha visto una incidencia muy baja en el seguimiento de estos pacientes. ${ }^{15}$

La principal limitación de este trabajo es que la experiencia es de un solo paciente, y la infraestructura insuficiente; sin embargo, el caso fue exitoso con los recursos disponibles y adaptados a una técnica quirúrgica con el propósito de preservar el intestino y evitar las complicaciones del síndrome de intestino corto secundario a las amplias resecciones.

\section{CONCLUSIÓN}

La enteroscopia transoperatoria con polipectomía es un método eficaz para el diagnóstico, prevención y tratamiento de una de las complicaciones principales de los pacientes con síndrome de Peutz-Jeghers. Con la aplicación de estas técnicas se previenen las resecciones intestinales múltiples y el tan temido síndrome de intestino corto como consecuencia de éstas. Su aplicación es segura en manos expertas y el índice de complicaciones es muy bajo; sin embargo, no debemos olvidar que este tipo de pacientes requiere seguimiento para prevenir recurrencias. El seguimiento puede ser por medio de enteroscopia con doble balón, cápsula endoscópica o resonancia magnética, para localizar el sitio del pólipo y resecarlo de manera oportuna.

\section{REFERENCIAS}

1. Peutz J. A very remarkable case of familial polyposis of mucous membrane of intestinal tract and accompanied by peculiar pigmentations of skin and mucous membrane. Nederl Maandschr Geneesk. 1921;10:134-6.
2. Jeghers H, McKusick VA, Katz KH. Generalized intestinal polyposis and melanin spots of the oral mucosa, lips and digits; a syndrome of diagnostic significance. $N$ Engl J Med. 1949;241:1031-6.

3. Korsse SE, van Leerdam ME, Dekker E. Peutz-Jeghers syndrome. Ned TijdschrTandheelkd. 2013;120:12-16.

4. Yu J, Jiang W. Acute intussusception and polyp with malignant transformation in Peutz-Jeghers syndrome: A case report. Oncol Lett. 2015;10(2):1008-10.

5. Cienfuegos JA, Baixauli J, Zozaya G, Bueno A, Arredondo J, Regueira FM, Angós R, Hernández-Lizoáin JL, Idoate MA. Peutz-Jeghers syndrome and duodeno-jejunal adenocarcinoma - therapeutic implications. Rev Esp Enferm Dig. 2009;101:875-9.

6. Hernan I, Roig I, Martin B, Gamundi MJ, Martínez-Gimeno $M$, Carballo $M$. De novo germline mutation in the serinethreonine kinase STK11/LKB1 gene associated with PeutzJeghers syndrome. Clin Genet. 2004;66:58-62.

7. Schreibman IR, Baker M, Amos C, McGarrity TJ. The hamartomatous polyposis syndromes: a clinical and molecular review. Am J Gastroenterol. 2005;100(2):476-90.

8. McGarrity TJ, Kulin HE, Zaino RJ. Peutz-Jeghers Syndrome. Am J Gastroenterol. 2000;95:596-604.

9. Oncel M, Remzi FH, Church JM, Connor JT, Fazio VW. Benefits of 'clean sweep' in Peutz-Jeghers patients. Colorectal Dis. 2004;6:332-5.

10. Baeza-Herrera C, García-Cabello LM, Nájera-Garduño HM, Sánchez-Fernández LA, Mora-Hernández F, OrtizZúñiga Al. Aspectos quirúrgicos de la invaginación secundaria al síndrome de Peutz-Jeghers. Cir Cir. 2005;73(2):91-5.

11. Hinds R, Philp C, Hyer W, Fell JM. Complications of childhood Peutz-Jeghers syndrome: implications for pediatric screening. J Pediatr Gastroenterol Nutr. 2004;39(2):219-20.

12. Van Lier MG, Westerman AM, Wagner A, Looman CW, Wilson JH, de Rooij FW, et al. High cancer risk and increased mortality in patients with Peutz-Jeghers syndrome. Gut. 2011;60:141-7.

13. Van Lier MG, Mathus-Vliegen EM, Wagner A, van Leerdam $M E$, Kuipers EJ. High cumulative risk of intussusception in patients with Peutz-Jeghers syndrome: time to update surveillance guidelines? Am J Gastroenterol. 2011 May;106(5):940-945.

14. Beggs $A D$, Latchford $A R$, Vasen HFA, Moslein $G$, Alonso A, Aretz S, Bertario L, Blanco I, et al. Peutz-Jeghers syndrome: a systematic review and recommendations for management. Gut 2010;59:975-86.

15. Wang $H$, Luo $T$, Liu WQ, Huang $Y, W u X T$ and Wang XJ. Clinical presentations and surgical approach of acute intussusception caused by Peutz-Jeghers syndrome in adults. J Gastrointest Surg. 2011;15: 2218-25.

16. Riegert-Johnson $D$, Roberts $M$, Gleeson FC, Krishna $M$, Boardman L. Case studies in the diagnosis and management of Peutz-Jeghers syndrome. Fam Cancer. 2011;10:463-8. 